\title{
La carga económica de la diabetes para los pacientes y sus familias en el Ecuador
}

\author{
Gladys Coello - Gómez'; David Gallegos - Coello² \\ Fecha de recepción: 7 de Diciembre 2017 \\ Fecha de aceptación: 7 de Diciembre 2018
}

\section{Resumen}

La diabetes mellitus tipo II, es la principal causa de muerte en el Ecuador y en general, a nivel mundial, constituye una preocupación para los gobiernos por los impactos económicos de la enfermedad en el presupuesto familiar y en el presupuesto estatal. La prevalencia de la enfermedad ha ido incrementando sin que existan mecanismos probados de políticas públicas que puedan frenar su avance. La presente investigación busca aportar con elementos orientadores, económicos y cuantificables, que sirvan de base para el diseño de programas públicos tendientes a prestar mayor atención a la inversión pública en la prevención, ya que una vez contraída la enfermedad, la carga económica de la diabetes para los pacientes, sus familias y el Estado bordea el $60 \%$ del salario básico mensual unificado; costo que genera que los pacientes mantengan tratamientos incompletos con consecuencias como problemas de salud más graves y costosos, muerte prematura, baja productividad, entre otros.

Palabras clave: Costos, diabetes, políticas públicas, prevención.

\section{Abstract}

Diabetes mellitus type II, is the main cause of death in Ecuador and in general, worldwide, is a concern for governments because of the economic impact of the disease on the family budget and the state budget. The prevalence of the disease has increased without the existence of proven public policy mechanisms that could slow down its progress. The present research seeks to contribute with guiding elements, economic and quantifiable, that serve as the basis for the design of public programs tending to pay greater attention to public investment in prevention, since once the disease is contracted, the economic burden of diabetes for patients, their families and the State it borders $60 \%$ of the unified monthly basic salary; cost that generates that patients maintain incomplete treatments with consequences such as more serious and expensive health problems, premature death, low productivity, among others.

Keywords: Costs, diabetes, public policies, prevention.

\section{Introducción}

Según Caro (2014) los costos económicos son considerados, como aquellos desembolsos de dinero por parte de quien padece la enfermedad y sus familiares o bien los costos en los que incurre el Estado para otorgar los servicios de salud correspondientes para quienes no puedan cotizar en el mercado de salud privado (todos estos son los costos directos). Quienes mueren a causa de una enfermedad crónica, incurren también en un costo que afecta a los ingresos, generalmente reflejado en el salario que dejan de percibir al fallecer prematuramente un familiar directo o a causa de la morbilidad, además de perder valioso tiempo de ocio y el aporte que realizaban sobre el trabajo en el hogar (conocidos como costos indirectos).

\section{Según el Banco Interamericano de Desarrollo (2015: 2):}

Los costos sociales son aquellos que reducen el bienestar total de la sociedad. En este caso, los costos médicos y salarios perdidos son claramente costos sociales porque son recursos que se podrían haber destinado a otros fines en la economía o a una actividad socialmente productiva. A pesar de que los costos del dolor y el sufrimiento no son verdaderos bienes o servicios que se intercambian en el mercado, los individuos están dispuestos a pagar para evitar el dolor, el sufrimiento y la pérdida de calidad de vida asociada con la condición de víctima de un delito. Por lo tanto, en medida en que la sociedad se ocupa del bienestar de las víctimas del crimen, estos costos también deberían considerarse costos sociales.

Stiglitz (2000) sostiene que el sector sanitario presenta imperfecciones por lo que el gobierno obligatoriamente debe intervenir para que la población con escaza capacidad de pago pueda acceder a servicios de salud. Entre las imperfecciones del mercado sanitario están la poca competencia, la información asimétrica que se da porque el paciente posee poca o ninguna información sobre el diagnóstico de la enfermedad y su tratamiento, la existencia de instituciones sin fines de lucro cuyo financiamiento proviene del Presupuesto del Estado o de donaciones del extranjero, pero que es canalizado por entes privados de manera discrecional, las aseguradoras médicas, entre otras; las cuales conducen a incrementar los costos de forma acelerada.

El informe del Instituto Nacional de Estadística y Censos del Ecuador, evidencia que la diabetes fue la primera causa de muerte en el país en los años 2011 y 2013 y la segunda causa en los años 2010, 2012 y 2014. En el año 2013, en el Ecuador murieron 4.695 personas a causa de la Diabetes Mellitus, siendo el género femenino, el más afectado, con 2.538 casos (INEC, 2014).

Cada año se diagnostican, en promedio 8.725 nuevos casos de diabetes. Para el año 2018, se estima que el número de enfermos de diabetes en el Ecuador bordeará las 573.122 personas.

Los costos de la diabetes son asumidos en parte por el Estado, quien brinda atención médica y entrega medicamentos a través de la red de servicios de salud pública y por otra parte, son enfrentados por el individuo y su familia cuando los servicios de salud públicos no son suficientes o encuentran dificultades para acceder a los mismos. Por tanto, la enfermedad trae consigo problemas que superan el costo directo del tratamiento de la enfermedad y que incide en otros campos de carácter social y económico como por ejemplo: la afectación a la productividad del paciente y de las personas cercanas al enfermo, el ausentismo laboral, entre otros.

El presente trabajo tiene como objetivo realizar un análisis del costo directo que enfrenta el Estado y las familias para atender a un paciente que sufre diabetes y proponer políticas públicas para focalizar el gasto público en los grupos más vulnerables de la sociedad ecuatoriana.

\section{Metodología}

La investigación se efectuó a través de un estudio exploratorio, descriptivo, transversal y no experimental, utilizando información disponible del Instituto Nacional de Estadística y Censos de Ecuador (INEC); así como fuentes bibliográficas, relacionadas con la investigación y publicadas por Organismos Mundiales en materia de salud. Es importante aclarar que para efectos del estudio y por tratarse de un abordaje de carácter sobre todo económico, para la presentación de los datos, se agrupa la información, prescindiendo de mostrar las estadísticas conforme la clasificación CIE-10 recomendada por la Organización Mundial de la Salud.

Para calcular el costo del tratamiento de la diabetes, se utilizará el método cuantitativo, empleando datos estadísticos del Instituto Nacional de Estadística y Censos de Ecuador (INEC), del Ministerio de Salud Pública (MSP) y organismos internacionales, relacionados con

Economista. Máster en Economía y Regulación de Servicios Públicos. Docente. Universidad de las Fuerzas Armadas-ESPE. Departamento Ciencias Económicas, Administrativas y de Comercio. Sangolquí- Ecuador. E-Mail: ggcoello@espe.edu.ec

EEconomista Independiente. Quito- Ecuador. E-Mail: drgallegosc77@outlook.com 
el número de enfermos, estimaciones del gasto sanitario en diabetes, tarifas públicas y privadas, entre otros. Paralelamente, esta información será complementada con una metodología cualitativa, la cual consiste en el análisis de la Guía Práctica Clínica del Ministerio de Salud Pública del Ecuador, de esta forma se podrá obtener mayor información sobre protocolos de atención en diabetes en el Ecuador acorde a las políticas de salud.

\section{Resultados}

En el periodo 2008-2014, en el Ecuador, la mortalidad a causa de la diabetes afectó más a las mujeres que a los hombres, como se puede observar en la Figura 1, el número de fallecimientos anuales a causa de la diabetes, entre los hombres se ha mantenido en un promedio de 1.931 muertes por año; mientras que en las mujeres el promedio de muertes anuales es mayor, ubicándose en 2.323.

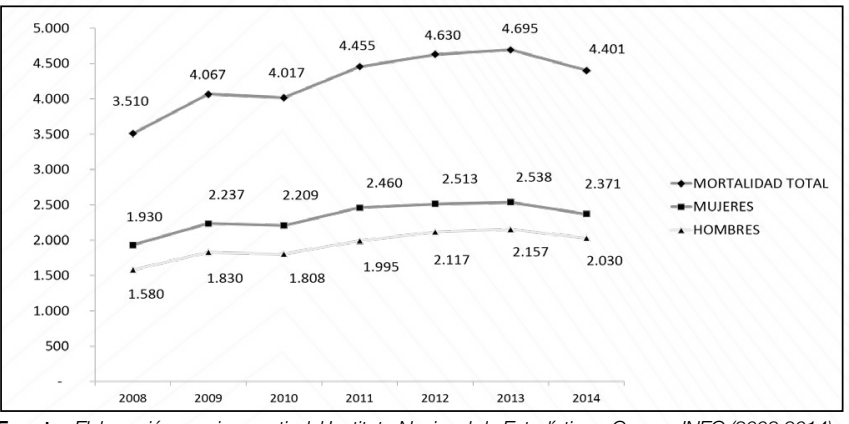

Fuente: Elaboración propia a partir del Instituto Nacional de Estadística y Censos INEC (2008:2014). Figura 1. Mortalidad por diabetes total y por género en el Ecuador. Periodo 2008-2014

"La prevalencia de diabetes en el área urbana es 3,2\% y en el área rural, $1,6 \%$. Las subregiones que presentan la prevalencia más alta de glucemia mayor a $126 \mathrm{mg} / \mathrm{dl}$ son Quito $(4,8 \%)$ y la Costa urbana $(3,8 \%)$ " (ENSANUT-INEC, 2013).

Según la Federación Internacional de la Diabetes (2013:141) se estima que en Ecuador la tasa de prevalencia de la diabetes es del $5,67 \%$ en la población de 20 a 79 años; en la Tabla 1 se ha calculado el número de enfermos diagnosticados con la enfermedad que en total, para el año 2018, se estima, ascenderían a 573.122 personas.

Tabla 1. Estimación del número de personas diagnosticadas con diabetes en el Ecuador, de 20 a 79 años de edad 2011-2018

\begin{tabular}{lcccc}
\hline Año & $\begin{array}{c}\text { Población total } \\
\text { Ecuador entre 20 a 79 } \\
\text { años }\end{array}$ & $\begin{array}{c}\text { Población con } \\
\text { diabetes }\end{array}$ & $\begin{array}{c}\text { Nuevos } \\
\text { diagnósticos por } \\
\text { año }\end{array}$ & $\begin{array}{c}\text { Prevalencia de diabetes en } \\
\text { población entre 20 a 79 } \\
\text { años }\end{array}$ \\
\hline $\mathbf{2 0 1 1}$ & 9.030 .776 & 512.045 & & $5,67 \%$ \\
$\mathbf{2 0 1 2}$ & 9.183 .902 & 520.727 & 8.682 & $5,67 \%$ \\
$\mathbf{2 0 1 3}$ & 9.336 .560 & 529.383 & 8.656 & $5,67 \%$ \\
$\mathbf{2 0 1 4}$ & 9.486 .135 & 537.864 & 8.481 & $5,67 \%$ \\
$\mathbf{2 0 1 5}$ & 9.637 .913 & 546.470 & 8.606 & $5,67 \%$ \\
$\mathbf{2 0 1 6}$ & 9.792 .120 & 555.213 & 8.744 & $5,67 \%$ \\
$\mathbf{2 0 1 7}$ & 9.948 .794 & 564.097 & 8.883 & $5,67 \%$ \\
$\mathbf{2 0 1 8}$ & 10.107 .974 & 573.122 & 9.026 & $5,67 \%$ \\
\hline
\end{tabular}

Fuente: Elaboración propia a partir del Instituto Nacional de Estadística y Censos INEC. Anuario de Estadísticas Vitales-Nacimientos y Defunciones (2011:2015)

Los costos directos generalmente incluyen gastos asociados a los tratamientos médicos, que van desde el diagnóstico, que puede implicar atención de emergencia y hospitalización, hasta el tratamiento de la enfermedad que debe ser continuo y permanente, para lo cual el paciente debe asistir a visitas médicas periódicas con facultativos especializados en diabetes, así como con médicos de otras especialidades como odontología, cardiología, oftalmología, psicología y educación nutricional, con el objetivo de evitar complicaciones o de ir controlando posibles daños en otros órganos del cuerpo como consecuencia de la enfermedad. Adicionalmente el paciente con diabetes debe someterse a exámenes clínicos habituales y consumir medicinas, insumos y suministros de forma diaria y de por vida.

El costo estimado del tratamiento de una persona que padece diabetes tipo II se ha calculado desde la perspectiva del sistema sanitario público y privado. Para el primer caso se utilizó la Guía Práctica Clínica y el tarifario del MSP y para el segundo las facturas de un paciente con diabetes que se hace atender en el servicio privado. En las Tablas 2 y 3 , se muestran los resultados, en el sistema sanitario público el costo anual asciende a US\$1.726,67 y el costo mensual a US\$143,89; mientras que en el sistema sanitario privado el costo anual es de US\$2.692,37 y el mensual de US\$224,36.

La diferencia de costos radica en que a nivel privado, al paciente se lo somete a una mayor cantidad de exámenes clínicos e interconsultas con especialistas; adicionalmente, a nivel del sector público no se provee al paciente de insumos necesarios para el control de la enfermedad como son el glucómetro y las tiras reactivas. Sin embargo, en esta materia, el 15 de noviembre de 2017, la Asamblea Nacional del Ecuador, emitió una Resolución en la que solicita al Ministerio de Salud Pública, dotar a la población de todos los insumos y medicamentos necesarios para el tratamiento de diabetes.

Tabla 2. Costo directo del tratamiento de la diabetes tipo I/ en el sistema sanitario público Año 2017

\begin{tabular}{|c|c|c|c|c|}
\hline \multicolumn{5}{|c|}{$\begin{array}{l}\text { La diferencia de costos radica en que a nivel privado, al paciente se lo } \\
\text { somete a una mayor cantidad de exámenes clínicos e interconsultas con } \\
\text { especialistas; adicionalmente, a nivel del sector público no se provee al } \\
\text { paciente de insumos necesarios para el control de la enfermedad como } \\
\text { son el glucómetro y las tiras reactivas. Sin embargo, en esta materia, } \\
\text { el } 15 \text { de noviembre de } 2017 \text {, la Asamblea Nacional del Ecuador, emitió } \\
\text { una Resolución en la que solicita al Ministerio de Salud Pública, dotar a } \\
\text { la población de todos los insumos y medicamentos necesarios para el } \\
\text { tratamiento de diabetes. }\end{array}$} \\
\hline \multicolumn{5}{|c|}{$\begin{array}{l}\text { Tabla 2. Costo directo del tratamiento de la diabetes tipo /l en el sistema sanitario público } \\
\text { Año } 2017\end{array}$} \\
\hline Código & Descripción & $\begin{array}{c}\text { Frecuencia } \\
\text { anual }\end{array}$ & $\begin{array}{c}\text { Valor Tarifario } \\
\text { MSP } \\
\text { US\$ }\end{array}$ & $\begin{array}{l}\text { Costo total } \\
\text { anual US\$ }\end{array}$ \\
\hline 340080 & Hemoglobina glicosilada & 4 & 7,89 & 31,56 \\
\hline 340025 & Hematocrito capilar & 2 & 1,55 & 3,10 \\
\hline 340026 & Hematocrito hemoglobina & 2 & 3,04 & 6,08 \\
\hline 360034 & AST (SGOT) & 2 & 2,17 & 4,34 \\
\hline 360035 & ALT (SGPT) & 2 & 4,53 & 9,06 \\
\hline 360039 & FOSFATAZA-ALKALINA & 2 & 3,23 & 6,46 \\
\hline 360161 & Cloro en orina & 1 & 2,48 & 2,48 \\
\hline 590049 & $\begin{array}{l}\text { GAMMAGRAFIA RENAL DTPA } \\
\text { FILTRACIÓN GLOMERULAR }\end{array}$ & 1 & 147,18 & 147,18 \\
\hline 360021 & Colesterol total & 2 & 3,29 & 6,58 \\
\hline 360147 & Colesterol LDL-HDL & 2 & 8,26 & 16,52 \\
\hline 360020 & Triglicéridos & 2 & 2,55 & 5,10 \\
\hline 360015 & Urea & 2 & 2,42 & 4,84 \\
\hline 360016 & Creatina & 4 & 3,48 & 13,92 \\
\hline 360182 & Microalbuminuria cuantitativa & 1 & 9,69 & 9,69 \\
\hline 290004 & TSH & 1 & 7,58 & 7,58 \\
\hline 360168 & Potasio en orina de 24 horas & 1 & 10,62 & 10,62 \\
\hline 360170 & Sodio en orina de 24 horas & 1 & 7,64 & 7,64 \\
\hline 380012 & EMO (uroanálisis de rutina) & 4 & 3,23 & 12,92 \\
\hline 340081 & Hemoglobina & 4 & 1,55 & 6,20 \\
\hline 99213 & Valoración endocrinológica & 4 & 13,50 & 54,00 \\
\hline 99213 & Valoración cardiológica & 1 & 13,50 & 13,50 \\
\hline 99213 & Valoración oftalmológica & 1 & 13,50 & 13,50 \\
\hline \multirow{5}{*}{99213} & Valoración odontológica & 2 & 13,50 & 27,00 \\
\hline & $\begin{array}{l}\text { Insulina NPH (acción Intermedia) } \\
\text { (3mensuales) }\end{array}$ & 36 & 16,50 & 594,00 \\
\hline & Metformina (90 tabletas mensuales) & 108 & 9,90 & 712,80 \\
\hline & & & TOTAL ANUAL & $1.726,67$ \\
\hline & & & TOTAL MENSUAL & 143,89 \\
\hline \multicolumn{5}{|c|}{$\begin{array}{r}\text { Fuente: Elaboración propia a partir de la Guía Práctica Clínica de la Diabetes Mellitus tipo II } \\
\text { del MSP (2017 y Tarifario del MSP (2014). }\end{array}$} \\
\hline
\end{tabular}

Fuente: Elaboración propia a partir de la Guía Práctica Clínica de la Diabetes Mellitus tipo /l del MSP (2017 y Tarifario del MSP (2014).

Tabla 3. Costo directo del tratamiento de la diabetes tipo II en el sistema sanitario privado

\begin{tabular}{lcrr}
\hline \multicolumn{1}{c}{ Descripción } & $\begin{array}{c}\text { Frecuencia } \\
\text { anual }\end{array}$ & $\begin{array}{c}\text { Tarifa Privada } \\
\text { US\$ }\end{array}$ & $\begin{array}{c}\text { Costo total } \\
\text { anual } \\
\text { US\$ } \$\end{array}$ \\
\hline Urea & 2 & 2,42 & 4,84 \\
Potasio en orina de 24 horas & 1 & 10,62 & 10,62 \\
Sodio en orina de 24 horas & 1 & 7,64 & 7,64 \\
Gasometría & 2 & 10,49 & 20,98 \\
Péptido C & 1 & 23,54 & 23,54 \\
TSH & 1 & 10,00 & 10,00 \\
FT4 & 1 & 10,00 & 10,00 \\
SPT (ALT) & 1 & 3,50 & 3,50 \\
Glucosa basal & 4 & 3,00 & 12,00 \\
PCR cuantitativo & 1 & 10,50 & 10,50 \\
Microalbuminuria cuantitativa & 1 & 15,00 & 15,00 \\
GAMMA G.T. & 1 & 5,00 & 5,00 \\
SGOT(AST) & 1 & 5,00 & 5,00 \\
Ácido úrico & 1 & 3,50 & 3,50 \\
Creatinina & 4 & 3,50 & 14,00 \\
Fructosamina & 4 & 10,00 & 40,00 \\
EMO & 4 & 3,00 & 12,00 \\
Hemoglobina glicosilada & 4 & 10,00 & 40,00 \\
Triglicéridos & 2 & 3,50 & 7,00 \\
Biometria hemática & 2 & 5,00 & 10,00 \\
HDL COLESTEROL & 1 & 6,00 & 6,00 \\
LDL COLESTEROL & 1 & 3,50 & 3,50 \\
Consulta Endocrinólogo & 4 & 60,00 & 240,00 \\
Consulta Cardiólogo & 1 & 60,00 & 60,00 \\
Consulta Oftalmólogo & 1 & 60,00 & 60,00 \\
\hline
\end{tabular}




\begin{tabular}{|c|c|c|c|}
\hline Consulta Odontólogo & 2 & 60,00 & 120,00 \\
\hline Consulta Nutricionista & 2 & 60,00 & 120,00 \\
\hline Consulta Sicólogo & 1 & 40,00 & 40,00 \\
\hline Glucómetro & 1 & 100,00 & 100,00 \\
\hline Tiras reactivas (1 diarias) & 365 & 0,75 & 273,75 \\
\hline $\begin{array}{l}\text { Insulina NPH (acción Intermedia) ( } 3 \\
\text { mensuales ) }\end{array}$ & 36 & 17,00 & 612,00 \\
\hline \multirow[t]{3}{*}{ Metformina (60 tabletas mensuales) } & 108 & 11,00 & 792,00 \\
\hline & & TOTAL ANUAL & $2.692,37$ \\
\hline & & TOTAL MENSUAL & 224,36 \\
\hline
\end{tabular}

Fuente: Elaboración propia a partir de facturas de tratamiento en servicio de salud privado Ecuador (2017).

La mayor parte del costo del tratamiento de la diabetes, se destina a medicamentos e insumos médicos para el control de las glicemias; en la Figura 2 se observa que dentro del $100 \%$ de costos del tratamiento de la diabetes tipo II, en el sistema sanitario privado, los exámenes clínicos representan el 10\%, el costo en las visitas médicas representa el $24 \%$, medicamentos e insumos médicos $52 \%$ y $14 \%$ respectivamente. En valores monetarios el costo mensual de los exámenes clínicos asciende en promedio a $\$ 22,89$, de las consultas médicas a $\$ 53,33$, de los medicamentos a $\$ 117,00$ y de los insumos a $\$ 31,14$.

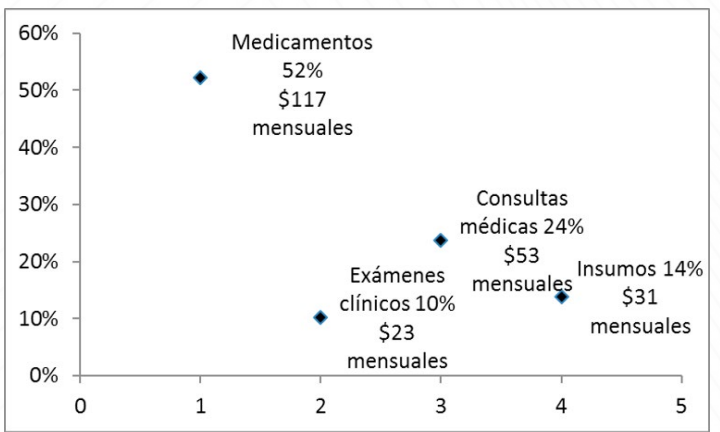

Fuente: Elaboración propia en base a los costos presentados en la Tabla 3. Figura 2. Distribución porcentual del costo directo privado en Ecuador por tratamiento de la diabetes 2017

\section{Conclusiones}

El costo directo del tratamiento de la diabetes en el Ecuador oscila entre \$143,89 y \$224,36 mensuales dependiendo si el tratamiento es provisto por el Estado, en el primer caso o si es costeado directamente por el paciente en el sistema de salud privado, en el segundo caso.

El salario mensual vigente en el Ecuador, en el año 2017, es de $\$ 375,00$, lo cual significa que una persona que padece diabetes y cuyo tratamiento se realiza en el sistema sanitario privado, debe dedicar alrededor del $60 \%$ de un salario básico mensual para atender su enfermedad; aspecto que coloca en situación de alto riesgo a aquellos enfermos de menores recursos económicos; quienes evidentemente en su elección como consumidores, siempre atenderán primero sus necesidades básicas y las de sus familias como alimentación, vivienda, transporte; difiriendo su atención de salud, lo cual traerá complicaciones del paciente, que en el futuro deberán ser afrontadas por el Estado.

Por otro lado, siendo que los medicamentos e insumos médicos juntos, representan un porcentaje que oscila el $66 \%$ del costo total del tratamiento mensual, estos no son provistos en su totalidad por el Estado; lo que significa que incluso cuando un paciente se atienda en el sistema sanitario público, este debe asumir parte del costo de su tratamiento.

Si bien la Asamblea Nacional del Ecuador, el 15 de noviembre de 2017, a razón de conmemorarse el Día Mundial de la Diabetes, emitió una Resolución en la cual reconoce que la diabetes es un problema de salud pública, que afecta a los individuos, a las familias y a la sociedad; dicha resolución no se ha elevado a un cuerpo normativo de mayor jerarquía y de aplicación obligatoria.

Durante la investigación, se encontraron una serie de dificultades, especialmente de carácter estadístico, por lo que se realizan dos recomendaciones en este ámbito: la primera está relacionada con la implementación de un Registro Único a nivel nacional de los enfermos de diabetes, lo cual permitirá realizar un mejor control de la evolución y pronóstico de la enfermedad, tal como ocurre en países desarrollados y en el caso de Latinoamérica en Chile; en donde tienen implementado este registro. El segundo tiene que ver, con conocer el nivel socioeconómico de la población en donde existe mayor prevalencia de la enfermedad, para lo cual se recomienda incluir en el próximo Censo de Población Económico una pregunta referente a que si algún miembro de la familia padece de una enfermedad crónica no transmisible y cuál es esta; esto permitirá realizar una mejor distribución del gasto sanitario público para la diabetes, asignando presupuesto focalizado para los grupos más vulnerables de la población y para programas de prevención a nivel nacional.

\section{Referencias}

Agencia de Evaluación de Tecnologías Sanitarias Instituto de Salud Carlos III - Ministerio de Economía y Competitividad. HIDALGO Á.; OLIVA, J.; RUBIO, M., ZOZAYA, N.; VILLORO, R.; GARCÍA, S., "Estudios de coste de la diabetes tipo 2: una revisión de la literatura". Madrid: Agencia de Evaluación de Tecnologías Sanitarias - Instituto de Salud Carlos III, febrero de 2015.

Gallegos, David (2017) "Valoración del costo social de enfermedades crónicas no transmisibles; Caso de la Diabetes Mellitus tipo II en el Ecuador, periodo 2008-2014". Disertación de grado, Facultad de Economía de la PUCE, Ecuador.

Federación Internacional de Diabetes (2011). Plan mundial contra la diabetes 2011-2021. Recuperado de: http://www.idf.org/sites/defaultffiles/attachments/GDP-Spanish.pdf

Federación Internacional de Diabetes (2013) Atlas de la diabetes de la FID. Sexta Edición. Versión online: www.idf.org/diabetesatlas.

García Soto Carlos Enrique (2007). El Costo de Vivir con Diabetes, Procuraduría Federal del Consumidor de México. Recuperado de: http://www. profeco.gob.mx/encuesta/brujula/bruj_2007/bol47_diabetes.asp

Gobierno de Chile (s.f.) QUALIDIAB - Chile: Revisión, Situación Actual y Brechas en la Región de Los Ríos. Recuperado de: http://www.academia. edu/16170313/QUALIDIAB_Chile_Revisi\%C3\%B3n_Situaci\%C3\%B3n_Actual_y Brechas_en_la_Regi\%C3\%B3n_de_Los_R\%C3\%ADos

Gómez, Oscar; Zapata, Pedro (2007). Contabilidad de Costos. Mc Graw Hill Interamericana, Tercera Edición, Colombia.

Instituto Nacional de Estadística y Censos (INEC) (2014), Diabetes y enfermedades hipertensivas entre las principales causas de muerte en el 2013. Recuperado de: www.ecuadorencifras.gob.ec/diabetes-y-enfermedades-hipertensivas-entre-las-principales-causas-de-muerte-en-el-2013/.

Instituto Nacional de Estadística y Censos (INEC) (2014), Encuesta Nacional de Ingresos y Gastos de los hogares urbanos y rurales 2011-2012. Recuperado de: http://www.inec.gob.ec/Enighur_/Analisis_ENIGHUR\%2020112012_rev.pdf, pp. 17, 23.

Hamis, M. (1995). Chapter I: Summary. Diabetes in America. 2nd Edition. USA. Javitt J. y Chiang. Y. (1995). Chapter 30: Economic Impact of Diabetes. Diabetes in America. 2nd Edition. USA.

Lucio Ruth, Villacrés Hilda y Henríquez, Rodrigo (2011). Sistema de Salud de Ecuador. Publicado en Scielo. Revista Salud Pública. México vol. 53 supl. 2. Cuernavaca ene. 2011. Recuperado de: $h$ ttp://www.scielo.org.mx/scielo. php?script=sci_arttext\&pid=S0036-36342011000800013

Novo Nordisk (2015) El desafío de la Diabetes en Chile. Recuperado de: http://www.novonordisk.cl/content/dam/Chile/AFFILIATE/www-novonordisk-cl/ Commons/Documents/Compendio\%20de\%20Diabetes_Chile_2015_es.pdf

Stiglitz, E. Joseph (2000) La Economía del Sector Público, Tercera Edición, España: Antoni Bosch, editor 